\title{
SPIRAL COLOUR MODEL: REDUCTION FROM 3-D TO 2-D
}

\author{
Frederic Garcia ${ }^{\star \dagger}$ Djamila Aouada ${ }^{\star}$ Bruno Mirbach ${ }^{\dagger}$ Björn Ottersten ${ }^{\star}$ \\ ${ }^{\dagger}$ A.E. Dept., IEE S.A. \\ \{frederic.garcia, bruno.mirbach\}@iee.lu \\ ${ }^{\star}$ SnT Centre, Univ. of Luxembourg \\ \{djamila.aouada, bjorn.ottersten\}@uni.lu
}

\begin{abstract}
We define a new reduced model to represent coloured images. We propose to use two components for a full definition of a colour instead of three. To that end we take advantage of the geometrical structure of the HCL conical colour space and approximate its circular base by a spiral. We thus write chroma as a function of hue. The resulting spiral is therefore defined by one parameter only. This parameter is then combined with luminance in order to represent all the colour information. Our experiments show that our proposed model ensures an accurate representation of coloured digital images. Furthermore, it preserves the perceptual properties of the original HCL representation.
\end{abstract}

Index Terms - Colour space, RGB model, colour transform, hue, chroma, luminance, Archimedean spiral.

\section{INTRODUCTION}

Colour analysis is a low-level operation which plays an important role in image processing algorithms such as classification, feature extraction or pattern recognition. Although there is a variety of colour spaces for colour representation, for instance RGB (red, green, blue), HSV/HSL (hue, saturation, value or luminance), or $\mathrm{L}^{*} \mathrm{a}^{*} \mathrm{~b} *$ (luminance $\mathrm{L} *$, opponent colour axes $\mathrm{a}^{*}$, and $\left.\mathrm{b}^{*}\right)[1,3,7]$, to name a few, all of them require three components to describe all the possible colours. In other words, all colour spaces are represented in three dimensions. This means that three independent channels are needed to process one coloured image. For instance, in the RGB model, a coloured image is represented with three images where each one contains the amount of each primary colour, i.e., red, green, and blue, at each image pixel. In many image processing techniques, these 3-D colour representations suppose a problem due to restrictions in processing time and memory requirements. This is why most image processing techniques operate on one grayscale image instead of three channels, where the grayscale image is defined as a linear combination of the red, green, and blue channels. This combination leads to a non-unique representation of the

F. Garcia was supported by the AFR Grant Scheme (Aides à la Formation-Recherche), managed by the National Research Fund Luxembourg (FNR). AFR Grant: TR-PHD BFR08-120. true colours, which may cause serious artefacts such as missing features, bad image matching, or non-detection of salient points.

Consequently, a more accurate processing of images, requires calling upon their true colours, and using three components. Indeed, most algorithms have their definitions extended to three channels such as bilateral filtering [4], image sharpening and denoising [2], image enhancement [10], colour image segmentation [8], etc. The choice of a colour space model over another is often motivated by using a more perceptually meaningful representation that usually defines a more discriminative distance between colours. Such a representation is shown to be conic as opposed to the cubic RGB model. Many models fall under the conic geometry such as HCV (hue, chroma, value), and lately the HCL colour space where $\mathrm{L}$ is for luminance [6].

In what follows, we propose to reduce the complexity of processing three channels by compactly storing the same information in just two channels. To that end, we exploit the geometrical structure of 3-D conical colour spaces and show how to accurately define one parameter as a function of another parameter defining the solid cone.

The organisation of the paper is as follows: In Section 2, we present the problem of reducing the dimensionality of a colour space. We then give the necessary background on the HCL model in Section 3. In Section 4, we define our new 2-D colour space and present its conversion from and to the RGB space. We present, in Section 5, the experimental results and evaluate approximation errors associated with our model. Finally, we give our conclusions and plans for future research in Section 6.

\section{PROBLEM STATEMENT}

Any colour can be fully defined by three components. Thus in the standard RGB model, for instance, a pixel on a coloured image is defined by its colour $\mathbf{p}=(r, g, b)$, where $\mathbf{p}$ is a point in the RGB cube shown in Fig. 2(a). The three axes $R$, $G$, and $B$ on the cube correspond to the primary red, green, and blue colours, respectively. Since today's vision applications require a fast processing of images, coloured images are usually converted to grayscale images. An image pixel is then defined by one scalar value $v$, which is a linear combination 


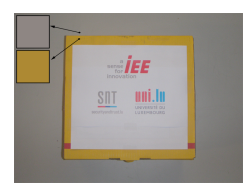

(a)

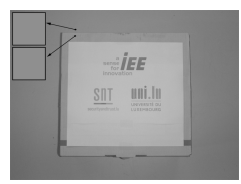

(b)

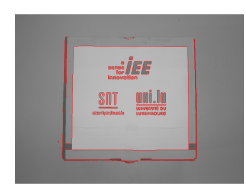

(c)
Fig. 1: Top view of a box on a table: (a) Coloured image. (b) Grayscale image. (c) Sobel filter applied on (b).

of the coordinates of p, i.e., $v=0.30 r+0.59 g+0.11 b$. As a result, many colours get collapsed to the same scalar value. Consequently, simple colour-based processing operations become inaccurate. The example in Fig. 1 illustrates the case where two distinct colours (shades of yellow and gray) become indistinguishable on a grayscale image. A classical Sobel filter fails in detecting the top edge of the box (Fig. 1(c)), while we can clearly see it on the coloured image in Fig. 1(a). The objective of this work is to define a colour model that is almost as reduced as the grayscale representation, but preserving all the colour information contained in 3-D spaces. In other words, we want to define a model that is in 2 dimensions, or less, and that is still reversible from and to the RGB colour cube.

\section{TRANSFORMATION FROM RGB TO HCL}

In addition to the above mentioned properties, our colour model should also bring in a perceptual meaning. This last property will be important when computing the distance between colours for pattern recognition purposes. To that end, we base our work on the conic HCL model shown in Fig. 2(c) [6]. We define in what follows the HCL model and relate it to the RGB cube as it will be a transition step in converting our proposed model to RGB space and vice versa. The projection of the RGB cube onto a regular hexagon $\Psi$ in the chromaticiy plane defines the chroma $C$ and hue $H$ related to $R, G$, and $B$ (Fig. 2b). Let $\mathbf{p}^{\prime}$ be the projection of a point $\mathbf{p}$ in the RGB cube on $\Psi$ and $o$ be the origin of $\Psi$. Geometrically, the chroma component $c$ along $C$, of $\mathbf{p}$, is the length of $\overrightarrow{\mathbf{o p}^{\prime}}$ relative to the maximal radius of $\Psi$ passing through $\mathbf{p}^{\prime}$. The hue component $h$ corresponds to the angle formed by $\overrightarrow{\mathbf{o p}^{\prime}}$ and $\overrightarrow{\text { or }^{\prime}}$, where $\mathbf{r}^{\prime}$ is the projection of the red colour $\mathbf{r}=(1,0,0)$ on $\Psi$. The luminance component $l$ is equal to $\left\|\overrightarrow{\mathbf{p p}^{\prime}}\right\|$. This is equivalent to $c=m_{1}-m_{2}$ and $l=\frac{1}{2}\left(m_{1}+m_{2}\right)$, where $m_{1}=\max (r, g, b)$, and $m_{2}=\min (r, g, b)$, and

$$
h=\left\{\begin{array}{lll}
\text { undefined } & \text { if } & c=0 \\
\frac{\pi}{3}\left(\frac{g-b}{c} \bmod 6\right) & \text { if } & m_{1}=r \\
\frac{\pi}{3}\left(\frac{b-r}{c}+2\right) & \text { if } & m_{1}=g \\
\frac{\pi}{3}\left(\frac{r-g}{c}+4\right) & \text { if } & m_{1}=b
\end{array}\right.
$$

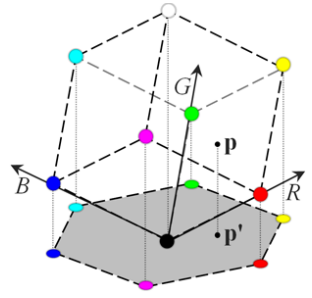

(a)

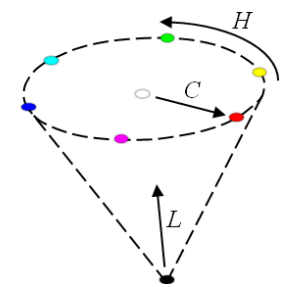

(c)

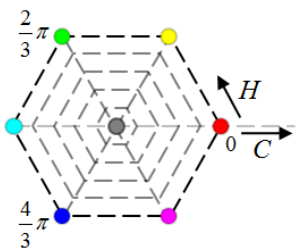

(b)

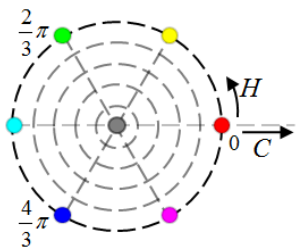

(d)
Fig. 2: (a) Cubic RGB model projected onto a regular hexagon in the chromaticity plane. From (b) to (d), warping from hexagons into circles. (c) Conic HCL model.

In what follows, we propose an approximation of the HCL space that only requires two parameters for colour description. There are alternative methods such as the proposed by Vahdat et al. [9], that describe the colour information by two or even one parameter. However, these methods are mainly related to data compression or codification and thus it is necessary to uncompress or decode the data to treat it.

\section{PROPOSED SPIRAL MODEL}

We propose to describe the colour information contained in the HCL space by approximating the HCL cone using two parameters, $\theta$ and $l$, instead of using the three coordinates $(c, h, l)$. We thus keep the luminance value $l$, and rewrite $c$ and $h$ as functions of a new variable $\theta$. Our key idea is to approximate the chromaticity disk with a spiral, as shown in Fig. 3. Specifically, we choose to use an Archimedean spiral [5] whose radial distance is defined as:

$$
r(\theta):=\frac{\mathrm{a} \cdot \theta}{2 \pi}
$$

where $\mathrm{a}$ is a constant distance between successive turns, and $\theta$ is the polar angle of the spiral, such that $\theta \in[0,2 \pi \mathrm{K}], \mathrm{K}$ being the total number of turns. We approximate the chromaticity disk by fitting the spiral to it, such that:

$$
h(\theta)=\theta+2 \pi k,
$$

where $k \in\{0,1, \cdots, \mathrm{K}\}$, and the $C$ - axis is uniformly sampled into $(\mathrm{K}+1)$ values $c_{k}$, with a step equal to a. We note that 


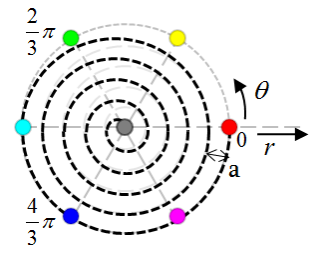

Fig. 3: Approximation of a disk by a spiral.

$c_{k}$ is dependent on the hue $h$, or equivalently of the angle $\theta$. Thus we define $c_{k}(\theta)$ as:

$$
c_{k}(\theta)=r(h(\theta))+\mathrm{a} \cdot k .
$$

By setting the spiral extremities as the starting point $\left(c_{0}, h_{0}\right)=$ $(0,0)$, and ending point $\left(c_{\max }, h_{\max }\right)=(1,0)$, and by replacing these values in (2) and (3), we find $a=1 / K$. In the continuous case, i.e., $\mathrm{K} \rightarrow \infty$, we may write

$$
c=r(\theta)=\frac{\theta}{2 \pi \mathrm{K}} \quad \Rightarrow \quad \theta=2 \pi \mathrm{K} \cdot c .
$$

Replacing (5) in (3), we find

$$
k=\operatorname{round}\left(\mathrm{K} \cdot c-\frac{h}{2 \pi}\right),
$$

with round $(\cdot)$ being a rounding function that assigns the nearest integer value to $k$. We may now define the transformation from $(c, h)$ to $\theta$ as follows:

$$
\theta=h-2 \pi \text { round }\left(\mathrm{K} \cdot c-\frac{h}{2 \pi}\right) .
$$

The inverse transformation from $\theta$ to $(c, h)$ is fully defined by (2), (3), and (4), with $k=\operatorname{round}\left(\frac{\theta-\theta \bmod (2 \pi)}{2 \pi}\right)$. Next step is the conversion from the recovered $c$ and $h$ values to the initial $(r, g, b)$. We compute an intermediate value $x=$ $c\left(1-\left|\left(\frac{3}{\pi} h\right) \bmod 2-1\right|\right)$ to be applied to the following system of equations:

$$
\left(r^{\prime}, g^{\prime}, b^{\prime}\right)= \begin{cases}(0,0,0) & \text { if } \mathrm{h} \text { is undefined } \\ (c, x, 0) & \text { if } 0 \leq h<\frac{\pi}{3} \\ (x, c, 0) & \text { if } \frac{\pi}{3} \leq h<\frac{2 \pi}{3} \\ (0, c, x) & \text { if } \frac{2 \pi}{3} \leq h<\pi \\ (0, x, c) & \text { if } \pi \leq h<\frac{4 \pi}{3} \\ (x, 0, c) & \text { if } \frac{4 \pi}{3} \leq h<\frac{5 \pi}{3} \\ (c, 0, x) & \text { if } \frac{5 \pi}{3} \leq h<2 \pi\end{cases}
$$

To obtain the point $\mathbf{p}=(r, g, b)$ from $\mathbf{q}=\left(r^{\prime}, g^{\prime}, b^{\prime}\right)$, we translate $\mathbf{q}$ in the $R, G$ and $B$ directions by the minimal distance $m_{2}$ defined in Section 3, i.e., $(r, g, b)=\left(r^{\prime}+m_{2}, g^{\prime}+\right.$ $\left.m_{2}, b^{\prime}+m_{2}\right)$. We note that the values of $m_{2}$ may be stored when extracting $c$ from $(r, g, b)$, or equivalently from $m_{2}=$ $\left(l-\frac{1}{2} c\right)$ (see Section 3).

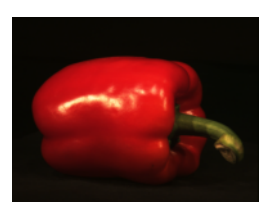

(a)

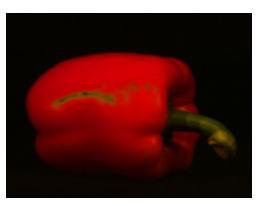

(b)

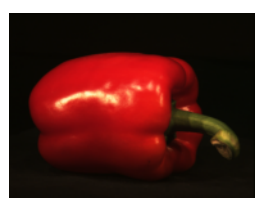

(c)
Fig. 4: (a) Original image (3-channels: $R, G, B$ ). (b) Recovered intermediate image (1-channel: $\Theta$ ). (c) Recovered full image (2-channels: $\Theta$ and $L$ ).

\section{EXPERIMENTAL RESULTS}

We start our experiments by giving an example of a coloured image represented using our proposed spiral model. We thus use the RGB image "pepper" from the Amsterdam Library of Object Images (ALOI) ${ }^{1}$. We first represent this image using one channel only, namely, the proposed spiral represented by $\Theta$, containing the values of $\theta$ as defined in (7). The visual result in Fig. 4(b) shows that the spiral model preserves well the colour information originally contained in the two channels $C$ and $H$. This result is already satisfactory for many colour-based operations. Moreover, it becomes suitable when luminance effect is to be removed. When combining $\Theta$ with $L$ components, i.e., using two channels, the recovered image, shown in Fig. 4(c), is exactly the same as the original one that was represented with three channels (Fig. 4(a)).

Next, we perform a global evaluation of the spiral model by testing 100 different coloured images of objects from the ALOI database. These images are initially in the RGB space. We transform them to the proposed $\Theta-L$ space. Figure 5 plots the root mean square error (RMSE) between the original and transformed images. The curves show the RMSE for K varying from 0 to 300. In addition, we plot also the RMSE for $H$ and $C$ separately. We see that chroma $C$ suffers much more than hue $H$ for small values of $\mathrm{K}$. This can be explained by

${ }^{1}$ http://staff.science.uva.nl/ aloi/

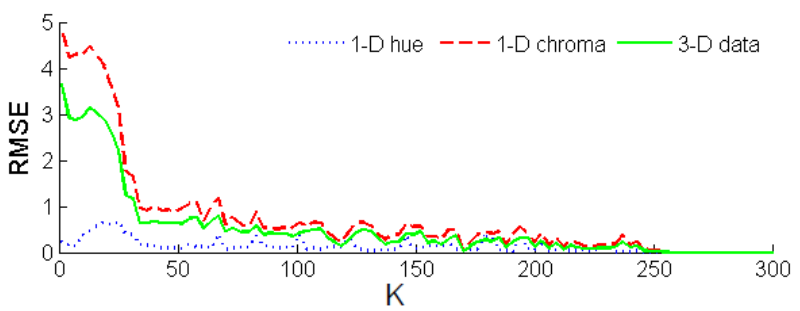

Fig. 5: RMSE between 100 images from the ALOI database and the transformed ones. 
the sampling performed by the spiral along the $C$-axis, and not on the angles $H$. Moreover, we note that as soon as $\mathrm{K}$ reaches 255 , the error drops to zero. This is due to the fact that discrete color components in digital images fall between 0 and 255. This means that there is no need to increase the sampling beyond $\mathrm{K}=255$, as it already ensures our model to represent digital images with high accuracy.

Since many image processing techniques are based on colour similarity measurements, we propose to check whether the spiral model $\Theta$ along with $L$ preserve the perceptual properties of the HCL space. We consider the image in Fig. 1, and use Euclidean distance to measure the dissimilarity between colours. For this evaluation, we take a yellow pixel from the box in Fig. 1a as a reference point $\mathbf{p}_{\mathbf{y}}$. We then compute the distance between $\mathbf{p}_{\mathbf{y}}$ and all other pixels. Fig. 6 shows the resulting distance images for each colour model, i.e., grayscale, RGB and $\Theta-L$. Fig. 6a confirms that grayscale images present almost no discrimination between clearly distinct colours. In contrast, the distance measurements in the RGB cube are much more accurate. However, we clearly see that the distances in the proposed spiral-luminance model correspond more to human perception, as shades of white and gray are clustered together, with respect to their dissimilarity with $\mathbf{p}_{\mathbf{y}}$. These results are confirmed by the distance histograms in Fig. 6. In the RGB case there are three clusters/modes, which means that similar colours are at different distances from the reference $\mathbf{p}_{\mathbf{y}}$. This could be the cause of many artefacts and errors.

\section{CONCLUSION}

We have demonstrated that HCL space, or any other 3-D colour space, can be accurately represented using two channels only. The dimensionality of any colour analysis problem can therefore be reduced from three to two dimensions while the information content remains fully preserved for an adequate sampling rate, i.e., $\mathrm{K}=255$ in the proposed spiral model formulation. We plan to further pursue our work to define one spiral for the approximation of the whole solid HCL cone. This may lead to colour space dimension reduction from 3-D to 1-D.

\section{REFERENCES}

[1] R. C. Gonzalez and R. E. Woods. Digital Image Processing. Prentice Hall, 2nd edition, 2002.

[2] T. Horiuchi, K. Watanabe, and S. Tominaga. Adaptive Filtering for Color Image Sharpening and Denoising. In Proceedings of ICIAPW'07, pages 196-201, 2007.

[3] N. P. Konstantinos and N. V. Anastasios. Color Image Processing and Applications. Springer, 1st edition, 2000.

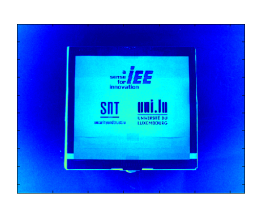

(a)

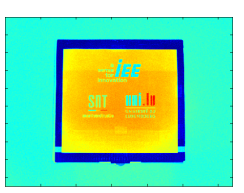

(c)

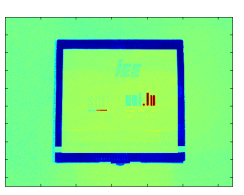

(e)

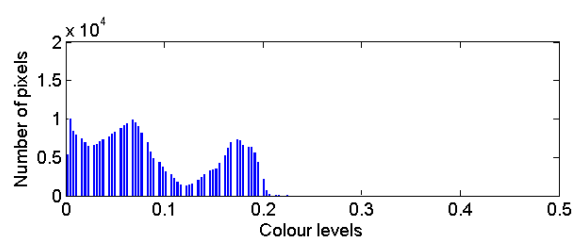

(b)

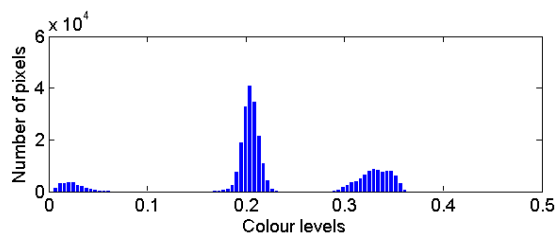

(d)

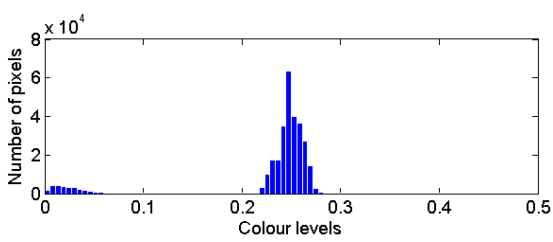

(f)
Fig. 6: Distance images and histograms between $\mathbf{p}_{\mathbf{y}}$ and the rest of the image. $(a, b)$ Grayscale image. $(c, d)$ RGB coloured image. (e,f) $\Theta-L$ image.

[4] J. Kopf, M. Cohen, D. Lischinski, and M. Uyttendaele. Joint bilateral upsampling. In Proceedings of ACM SIGGRAPH'07, page 96, 2007.

[5] E. H. Lockwood. A Book of Curves. Cambridge University Press, 1st edition, 1967.

[6] M. Sarifuddin and R. Missaoui. A new perceptually uniform color space with associated color similarity measure for contentbased image and video retrieval. In Proceedings of Multimedia Information Retrieval Workshop, pages 3-7, 2005.

[7] G. Sharma and H. Trussell. Digital Color Imaging. IEEE Transactions on Image Processing, 6(7):901-932, 1997.

[8] O. Sojodishijani, V. Rostami, and A. Ramli. Real Time Colour Image Segmentation with Non-Symmetric Gaussian Membership Functions. In Proceedings of CGIV'08, pages 165-170, 2008.

[9] A. Vahdat and M. S. Drew. Colour from grey by optimized colour ordering. In Proceedings of $\mathrm{CIC}^{\prime} 10$, pages 17-21, 2010.

[10] D. Xinghao, W. Xinxin, and X. Quan. Color image enhancement with a human visual system based adaptive filter. In Proccedings of IASP'10, pages 79-82, 2010. 